\title{
Relationship between electrical and mechanical properties of motor units
}

\author{
T Vogt, W A Nix, B Pfeifer
}

\begin{abstract}
In six healthy volunteers, the size of the motor unit action potential (MUAP) was related to its contractile behaviour. Electrical activity was determined by measuring amplitude and area of the potential, using the macro electromyography (EMG) technique. The mechanical response was characterised by contraction time, twitch force and relaxation time, recorded with the spike triggered averaging technique. With increasing tension of the whole muscle corresponding to an increasing threshold, the force of single units enlarged exponentially compared to their EMG. There was only a weak relationship between contraction time and electrical activity and none between EMG and relaxation time.
\end{abstract}

Conventional needle electromyography restricts the uptake area to a hemisphere with a radius of $1 \mathrm{~mm}$. The average diameter of a normal motor unit is about $5-10 \mathrm{~mm}$. Thus only part of the muscle fibres contribute to the spike part of the EMG. More information about the size of a motor unit is obtained with the macro EMG technique. ${ }^{12}$ The aim was to record intramuscularly with the large uninsulated surface of the electrode shaft the activity of all fibres within the uptake area and to record the activity of a single muscle fibre by a single fibre electrode within the same cannula. The contribution of one individual motor unit to the whole activity is obtained when the single fibre EMG triggers the averaging of the signal picked up by the large uptake surface. The area of the rectified macro potential represents the electrical activity of all fibres belonging to one unit. Configuration of the single fibre potential gives information about the fibre density. ${ }^{3}$

Since force is the functional parameter of the motor unit size, the study was focused on potential size measurements with simultaneous recordings of force. This relationship has not been considered in detail previously. Early studies recorded force and EMG from the whole muscle and from theoretical treatment of the results, it was concluded that a relation exists between tension and the size of the potential of single units. ${ }^{4}$ The particular aim of this study was to evaluate parameters of EMG previously used to determine unit size in clinical practice with direct measurements of force.

\section{Methods}

Single motor units have been studied from the first dorsal interosseus muscle in the hand of six normal subjects, ranging from 25 to 59 years of age. This muscle contains all four different types of muscle fibres; its limited number of units represent a broad range of size. It has been used for previous studies of motor unit behaviour, which allows for comparisons. ${ }^{5-8}$

\section{EMG recordings}

Motor unit action potentials were recorded with the macro EMG technique. The aim was to record electrical activity of a muscle with a needle electrode using a large recording surface relative to a remote subcutaneous concentric needle electrode, simultaneously with the EMG activity of one or two muscle fibres. The macro needle consists of a steel cannula in which the $15 \mathrm{~mm}$ uninsulated tip serves as the recording surface. The uptake area is large enough to record the activity of all muscle fibres within one motor unit and is not significantly influenced by the relative distance of single muscle fibres from the electrode. A side port, $7.5 \mathrm{~mm}$ behind the tip, exposes the single fibre electrode which has the bared cannula as reference. Within an uptake area with a radius of about $270 \mathrm{um}$, this electrode records action potentials of one or two muscle fibres. The single fibre EMG (SFEMG) is amplified, filtered (bandpass of $500 \mathrm{~Hz}$ and $10 \mathrm{kHz}$ ) and displayed on an oscilloscope with a sweep velocity of $2 \mathrm{~ms} / \mathrm{cm}$. The macro EMG signal requires a bandpass of $5 \mathrm{~Hz}$ to $10 \mathrm{kHz}$ and is displayed on a second channel with a slower sweep velocity of $8 \mathrm{~ms} / \mathrm{cm}$ to allow resolution of long duration potentials (Amplifier Medelec MS 6, Medelec Ltd, United Kingdom). The SFEMG is used to trigger the averaging process using a personal computer (Med 3000 System, Fa Fischer, Medical Computer System, West Germany).

Only those single fibre EMG signals were accepted that had an amplitude of at least 200 $\mu \mathrm{V}$ and a rise time less than $300 \mu \mathrm{s}$. After about 100 averaging steps, the shape of the potential and the baseline show no further change. The digitised averaged signals were stored on disc.

\section{Force measurement}

To record twitch force, the right forearm of the subject was comfortably rested and fixed in a plaster cast moulded to the arm up to the elbow. A strain gauge (F Fleck, Department of Pharmacology, University of Mainz) with a sensitivity range of $1 \mathrm{mN}$ to $20 \mathrm{~N}$ was positioned against the proximal interphalan- 


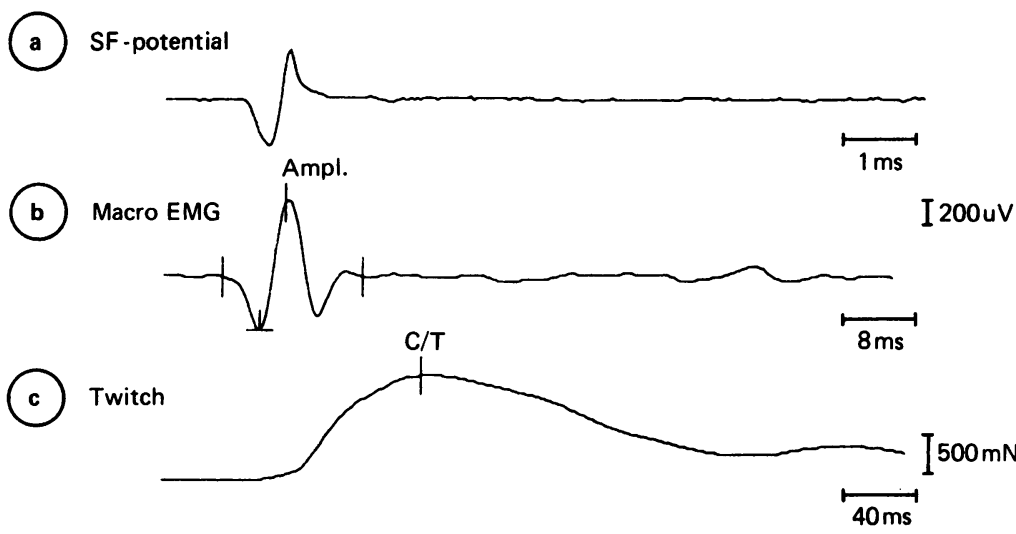

Figure 1 Recordings from the FDI muscle. The upper trace shows the single fibre EMG, the middle trace the macro EMG and the lower the twitch of one motor unit. Note different time scales for $a, b$ and $c$.

geal joint of the index finger. Subjects were asked to activate the first dorsal interosseus muscle with visual and auditory feed back. The motor units were activated within different threshold levels and the discharge rate was kept as low as possible. Single units were selected by altering the triggering threshold.

Up to 50-100 sweeps were averaged until onset, offset, amplitude and configuration did not change from visual inspection. We used less averaging steps than described in other studies 5 because we recorded single contractions averaged from brief rather than continuous activation. Furthermore, it has been described that partial fusion of a unit's twitch profile can seriously distort the twitch parameters. ${ }^{910}$ The SFEMG was used to trigger the computer which averaged the macro potential as described earlier and the DC coupled force simultaneously. The filter settings for the DC amplifier were 0 and $100 \mathrm{~Hz}$.

We also compared the twitches, recorded with the spike triggered averaging technique with the results of contractile measurements obtained from intramuscular microstimulation in another group of subjects.

In this technique, the forearm is rested as described for the spike triggered averaging technique. A tungsten electrode for stimulation and a concentric needle electrode to record the action potential were inserted into the muscle. Single motor axons were stimulated following the all or nothing principle. The examination was.performed on 10 healthy subjects between 18 and 69 years of age and 62 units were recorded. Simultaneous recording of macro potential was not possible because the macro signal was contaminated by the stimulus artefact.

Figure 2. Distribution of contraction times amon motor units from FDI.
Data analysis

The following parameters were measured: contraction time (CT), defined as the time between onset of the SF potential and peak force; half relaxation time ( $\left.\frac{1}{2} \mathrm{RT}\right)$; twitch force (PT); area of the macro potential; amplitude of the macro potential. Onset and offset of the potential as well as beginning and twitch maximum were defined by visual inspection for the baseline. Amplitude, area and half relaxation time were calculated by computer.

\section{Results}

Figure 1 shows a typical recording of twitch forces and macro EMGs of 50 motor units of the first dorsal interosseus muscle, where the single fibre potential (a) which served as trigger signal for (b) the macro potential and (c) the twitch are displayed on different time scales.

Twitch contraction times are unimodally distributed (fig 2) with a range between 36 and $140 \mathrm{~ms}$. Similar data were obtained with the method of intramuscular microstimulation with only a slight shift in contraction time.

Half relaxation time of the twitch correlates with CT $(R=0,45)$ but less with twitch amplitudes (fig 3 a, b). PT showed a skewed distribution (fig 4) with a range between $6 \cdot 3$ $\mathrm{mN}$ and $3.5 \mathrm{~N}$ and peaking between 50 and 100 $\mathrm{mN}$. About two thirds of the units are in the range between 10 and $100 \mathrm{mN}$, and the median of $39,1 \mathrm{mN}$ is the more appropriate value. Similar values between $1 \mathrm{mN}$ and $1.5 \mathrm{~N}$ were found by others with a comparable technique. ${ }^{5}$ Data obtained by intramuscular microstimulation demonstrate the same distribution (fig 5).

The weak relationship between twitch force and contraction time corresponds to previous findings. ${ }^{6}$ Area and amplitude characterise the macro potential. Duration is not considered in this study as it is sometimes very difficult to define onset and offset exactly. Longer duration parameters have only a slight influence on
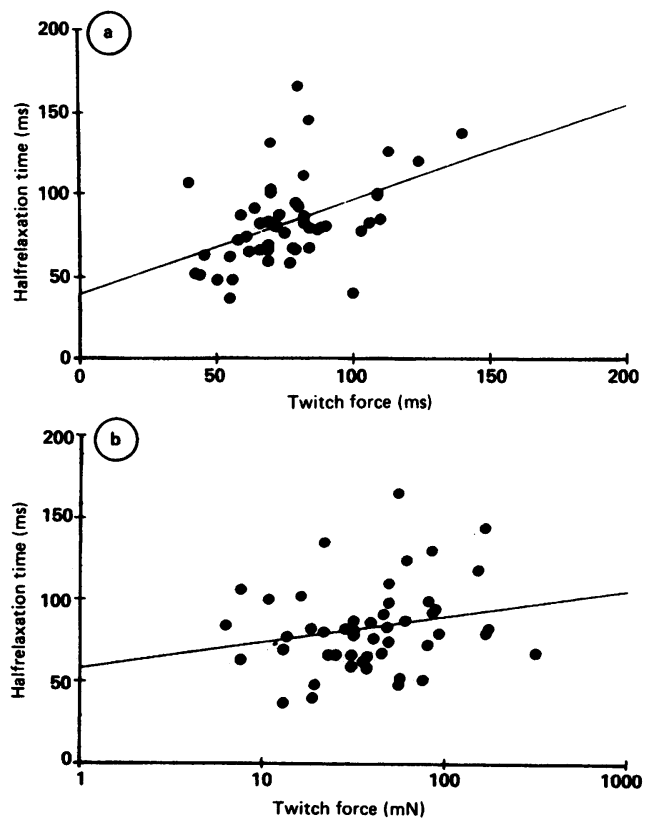

Figure 3 Correlation between half relaxation time and $(A)$ contraction time and $(B)$ twitch force. Contraction time shows a correlation with half relaxation time; twitch force is not related with relaxation. 
Figure 4 Histograms showing the distribution of (A) twitch forces, (B) amplitudes and (C) areas of the macro EMG. EMG amplitudes are skewed distributed, the macro EMG areas have a Twitch forces and macro normal distribution.
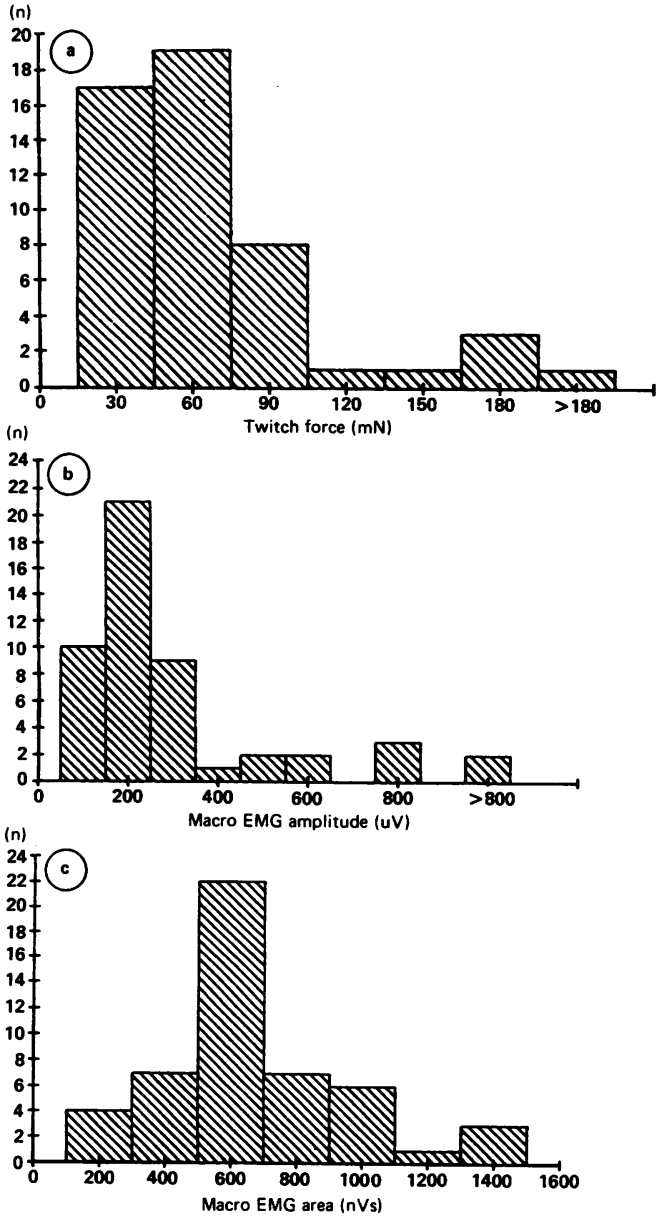

area measurements. ${ }^{11}$ The size of macro motor unit potentials are scattered over a wide range, the areas between $122 \mathrm{nVs}$ and $1350 \mathrm{nVs}$, the amplitudes between $40 \mathrm{uV}$ and $1280 \mathrm{uV}$. Areas are normally distributed, whereas the amplitudes show a skewed distribution with a predominance of smaller values (fig $4 \mathrm{~b}, \mathrm{c}$ ).

The single fibre potential which was used as the trigger signal, always had normal configuration, and no increased fibre density was found. The relationship between twitch force and electrical activity of individual motor units in healthy subjects relies on the data of 50 units (fig 6). PT are plotted in a logarithmic scale because of the large range of values.

There was a good correlation between PT and macro EMG area (Coefficient $=0.84$, $\mathrm{p}<0.001$ ). With increase of macro EMG area, twitch tension increases exponentially. There is a power correlation between PT and macro EMG amplitude (Coefficient $=0.63$, $\mathrm{p}<0.001)$.

Figure 5 Histograms showing the distributions of motor unit twitch forces obtained by using spike triggered averaging technique in our six subjects (hatched box) and intramuscular microstimulation (blank box) in another population of 10 subjects. Both techniques show a similar distribution of twitch forces.

\section{Discussion}

In this study, a combination of macro EMG and spike triggered averaging has been used to characterise motor unit size. Since Henneman demonstrated that the recruitment and firing properties of motor units are determined by their size ${ }^{12}$ and according to the results of Herdmann et $a l^{13}$ who found a clear dependence of motor unit action potential size on recruitment threshold, thus indicating that the size principle can be extended to EMG properties as well, we expected a dependence of twitch force on potential size.

Early theoretical studies on the relation between twitch force and electrical potential in a muscle, assumed that the EMG amplitude of a single unit would increase less rapidly than its twitch tension. ${ }^{14}$ A few authors reported a relatively larger increase in potential size. ${ }^{4}$ These were, however, indirect measurements, based on the increase of the surface EMG during a contraction. Milner-Brown and his

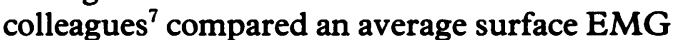
of a single unit with its twitch tension and found a nonlinear relationship, indicating a smaller relative increase of potential size.

Since surface EMG has the disadvantage that the distance between the muscle fibres and the electrode varies so that amplitude depends on the location of the unit within the muscle ${ }^{15}$ we used macro EMG. In this technique the distance between the fibres and the macro needle does not greatly affect the potential. ${ }^{1}$

We found a good correlation between PT and macro EMG size, where force increases more rapidly than electrical activity of single units during an isometric contraction of low threshold units. For the two parameters of macro EMG measurements, the area has a better correlation than the amplitude. The distribution of the amplitude values indicates, that in the small units, the correlation also
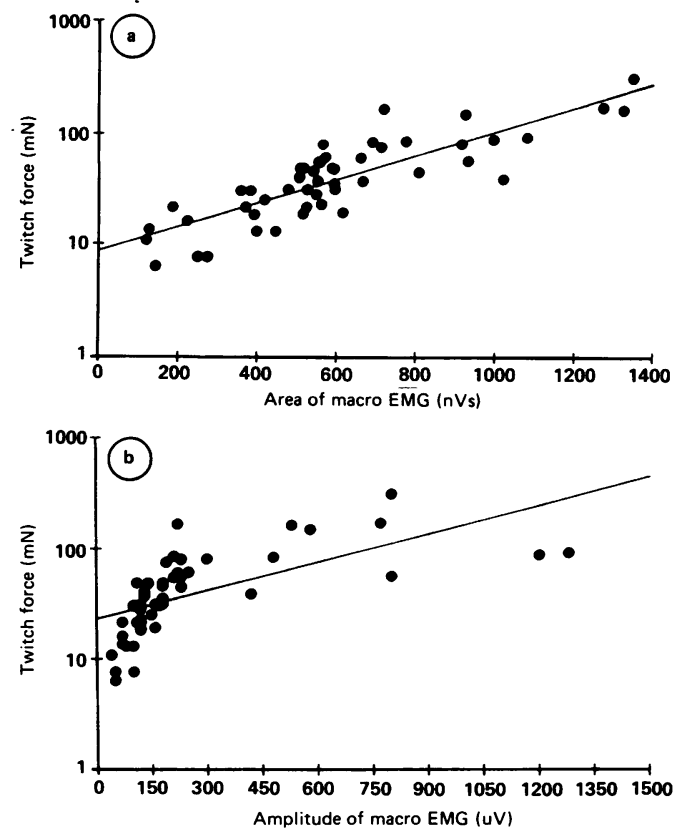

Figure 6 Correlation between twitch force of motor units and $(A)$ macro EMG area and $(B)$ macro EMG amplitude. Twitch forces are scaled in a logarithmic manner. The size of the EMG potential increases seven times, the twitch force increases approximately 20 times. 
improves. In larger units, however, the amplitude increases more than area. Since area depends on amplitude, duration and shape, this can only be explained by differences in temporo-spatial dispersion of the single fibre activities during the formation of the compound potential.

This result seems to be contradictory to the findings that the surface EMG varies linearly with the force generated by the whole muscle at a constant length. ${ }^{16}$ The generated force is, however, a result of recruitment and rate coding. ${ }^{17-19}$ At the beginning of a contraction, at low force levels, twitch force is mainly increased by recruiting more units with small forces. After large units have been recruited, frequency coding comes into play to further increase muscle force. Due to these two factors, the compound surface EMG varies linearly with the total force. The force of a single unit is, however, a product of the number of fibres and its total cross sectional area. ${ }^{20}$

Twitch tension will increase with the square of muscle fibre diameter, whereas voltage will only increase linearly because of potential spread. ${ }^{14}$ Given these considerations, it is logical, that the units behave in the same way.

The area of macro potentials ranged from $122 \mathrm{nVs}$ to $1350 \mathrm{nVs}$, corresponding fairly well to the normal values of the extensor digitorum communis muscle (EDC). ${ }^{11}$ Area and amplitude of the macro potential are correlated. Contraction parameters were measured to characterise these macro EMG values within this large range. These parameters show that the contraction speeds were unimodally distributed; no clear division between fast and slow twitch motor units was found. These results agree with previous studies using spike triggered averaging. ${ }^{1521}$ Similar data is obtained with intramuscular microstimulation. ${ }^{56}$

Half relaxation time showed a weak correlation to the contraction times and no correlation was found to twitch force. The reason is possibly a greater variation of half relaxation time and a contamination with other units in that late period of contraction, whereas the early time course near the trigger point can be sharply differentiated by the averaging process.

Twitch force had a skewed distribution with small units more common than larger ones. The number of large units decreased in a nearly exponential fashion compared to the linear increase of potential size of the single units. This confirms our results of an exponential distribution of twitch force measured by intramuscular microstimulation and agrees with the results of Milner-Brown et al. ${ }^{14}$

We conclude that measurement of EMG, force and twitch time provides a comprehensive electrophysiological characterisation of the motor unit.

We thank Tessa Gordon and Joanne Totosy de Zepetnek, University of Alberta, Edmonton, Canada for their helpful comments on the manuscript.

This study was supported by the Deutsche Forschungs Gemeinschaft.

1 Stalberg E. MACRO EMG, a new recording technique. $J$ Neurol Neurosurg Psychiatry 1980;43:475-82.

2 Stalberg E. New EMG methods to study the motor unit. Electroencephalogr Clin Neurophysiol 1987;39(suppl): 38-49.

3 Stalberg E, Thiele B. Motor unit fibre density in the extensor digitorum muscle. J Neurol Neurosurg Psychiatry 1975;38:874-80.

4 Bigland B, Lippold OC. The Relation between force, velocity and integrated electrical activity in human muscle. J Physiol 1954;123:214-24.

5 Dengler R, Stein RB, Thomas CK. Axonal Conduction Velocity and Force of Single Motor Units. Muscle Nerve 1988;11:136-45.

6 Mayer RF, Young JL. Physiological properties of muscle units in man. Trans Am Neurol Ass 1979;104.

7 Milner-Brown HS, Stein RB, Yemm R. The contractile properties of human motor units during voluntary isometric contractions. J Physiol 1973;228:285-306.

8 Stephens JA, Usherwood TP. The mechanical properties of human motor units with special reference to their fatiguability and recruitment threshold. Brain Res 1977 125:91-7.

9 Andreassen S, Bar-on E. Estimations of motor unit twitcher. IEEE Trans Biomed Eng 1985;30:742-8.

10 Calancie B, Bawa P. Limitations of the spike triggered averaging technique. Muscle Nerve 1986;9:78-83.

11 Pfeifer B, Nix WA, Vogt T. MAKRO EMG: Methodik und probleme der normwerterstellung. $Z E E G E M G 1988$; probleme

12 Henneman E, Olsen CB. Relations between structure and function in the design of human skeletal muscle. Neurophysiol 1965;28:581-98.

13 Herdmann H, Büdingen HJ, Reiners K, Berger W, Freund HJ. Die abhängigkeit der aktionspotentialamplitude motorischer einheitern von der rekrutierungsschwelle. $Z$ EEG EMG 1986;17:140-5.

14 Milner-Brown HS, Stein RB. The relation between the surface EMG and muscular force. $J$ Physiol 1975;246: 549-69.

15 Butler RP, Ball RD, Albers JW. Effect of electrode type and position on motor unit action potential configuration Muscle Nerve 1982;5:S95-7.

16 Lawrence HJ, De Luca CJ. Myoelectric signal versus force relationship in different human muscles. $J$ Appl Physiol relationship in

17 Freund HJ. Motor units and muscle activity in voluntary motor control. Physiol Rev 1983;63:387-436.

18 Milner-Brown HS, Stein RB, Yemm R. The orderly recruitment of human motor units during voluntary isometric contraction. J Physiol 1973;230:359-70.

19 Monster AW, Chan H. Isometric force production by moto units of extensor digitorum muscle in man. J Neurophysio 1977;40:1432-43.

20 Stein RB, Gordon T, Totosy de Zepetnek J. Mechanisms for respectifying muscle properties following reinnervation. In: Binder MD, Mendell IM, eds. The Segmental Motor System. Oxford: Oxford Press (in press).

21 Stein RB, French AS, Mannard A, Yemm R. New methods for analysing motor function in man and animal. Brain Res 1972;40:187-92. 